\title{
Haga usted el diagnóstico \\ Parte II \\ Penfigoide ampolloso
}

\section{Juan David Ruiz Restrepo'; Melissa Alejandra Quintero Picón²; Laura Trujillo Gaviria³}

\section{RESUMEN}

Es una enfermedad ampollosa subepidérmica autoinmune de presentación infrecuente, originada por la presencia de autoanticuerpos que se dirigen contra componentes de la membrana basal epidérmica, conocidos como BPAG1 y BPAG2. Se presenta principalmente en adultos mayores y está asociado a una alta morbilidad y mortalidad. Clínicamente se caracteriza por la aparición de ampollas tensas sobre placas eritematosas, edematosas y pruriginosas. Para el diagnóstico, es importante la correlación de la clínica, evaluación histopatológica y el estudio por medio de inmunofluorescencia. En caso de presentaciones atípicas, el diagnóstico puede convertirse en un reto médico. El tratamiento se basa en el uso de inmunosupresores, principalmente los esteroidesm.

PALABRAS CLAVE: penfigoide ampolloso; ampolla subepidérmica.

\section{BULLOUS PEMPHIGOID}

\section{SUMMARY}

It is an autoimmune subepidermal bullous disease of infrequent presentation, caused by the presence of autoantibodies that are directed against epidermal basement membrane components, known as BPAG1 Y BPAG2. It occurs mainly in older adults associated with high morbidity and mortality. Clinically it is characterized by the appearance of tense blisters on erythematous, edematous and pruritic plaques. For the diagnosis it is important the correlation of the clinic, histopathological evaluation and the study by immunofluorescence. In case of atypical presentations, the diagnosis can become a medical challenge. The treatment is based on the use of immunosuppressants, mainly steroids.

KEY WORDS: Bullous pemphigoid; Subepidermal blister.

1. Patólogo, Docente de Dermatopatología, Universidad de Antioquia, Medellín, Colombia. Patólogo, SURA Ayudas Diagnósticas, Medellín, Colombia. ORCID https://oooo-0002-3000-2942

2. Residente de Patología, Universidad de Antioquia, Medellín, Colombia. ORCID https://0000-0002-4093-9704

3. Residente de Dermatología, Universidad de Antioquia, Medellín, Colombia. ORCID https://oooo-0002-8053-156X

Correspondencia: Juan David Ruiz Restrepo; email: juandavidruizrpo@gmail.com

Recibido: 06/02/20; aceptado: 30/04/20

Cómo citar: Ruiz-Restrepo JD, Quintero-Picón MA, Trujillo-Gaviria L. ¿Reconoce esta clave diagnóstica? Rev Asoc Colomb Dermatol. 2020;28(1): 8-14. DOI:

Financiación: ninguna, conflictos de interés: ninguno 


\section{GENERALIDADES}

Enfermedad ampollosa subepidérmica de origen autoinmune, descrita como la enfermedad ampollosa más frecuente en muchos estudios. Aunque no representa per se un alto riesgo para la vida del paciente, se asocia con alta morbilidad y mortalidad, debido a la frecuente relación con comorbilidades y efectos adversos al tratamiento. Ha presentado una incidencia creciente en los últimos años, reportada de 4 a 22 casos por millón de habitantes al año. Es más común en personas mayores de 60 años y presenta un leve predominio en mujeres.

\section{ETIOLOGÍA}

Se origina debido a la formación de autoanticuerpos dirigidos contra algunas de las proteínas estructurales de los hemidesmosomas, principalmente, la proteína BP180 y BP230. Se ha asociado a la exposición de algunos medicamentos, enfermedades neurológicas o neoplasias. La unión de los anticuerpos con los antígenos lleva a la activación de la cascada inflamatoria mediada por el complemento. Esto resulta en el reclutamiento de células inflamatorias que liberan enzimas proteolíticas que destruyen la unión dermoepidérmica.

\section{MANIFESTACIONES CLÍNICAS}

Clínicamente, las lesiones empiezan como placas eritematoedematosas, muy pruriginosas, que pueden durar días o incluso meses y que pueden tener un aspecto urticarial o prurigoide. Sobre estas lesiones aparecen ampollas tensas de contenido seroso o hemorrágico. El compromiso es simétrico y principalmente localizado en el tronco y en las zonas flexurales. Las ampollas resuelven sin dejar cicatriz. Hasta en un 30\% de los casos pueden presentarse formas localizadas de la enfermedad, como en las piernas, perigenital, periostomal o sitios de trauma. También se conocen algunas formas no ampollosas del penfigoide.

Para el diagnóstico, es importante tomar una biopsia de una ampolla y otra de piel perilesional, para el estudio de inmunofluorescencia directa (IFD), con técnica de hendidura salina. En la histopatología podemos encontrar una hendidura subepidérmica con infiltrado inflamatorio mixto con predominio de eosinófilos. En la IFD se encuentran depósitos lineares de IgG y C3 en la unión dermoepidérmica. Por medio de la hendidura salina y la marcación en el techo de la ampolla podemos diferenciar esta enfermedad de otras enfermedades ampollosas subepidérmicas.

\section{DIAGNÓSTICOS DIFERENCIALES CLÍNICOS}

Urticaria, eritema multiforme, epidermólisis pénfigo vulgar, dermatitis herpetiforme, dermatosis IgA lineal y lupus ampolloso.

\section{DIAGNÓSTICOS DIFERENCIALES HISTOLÓGICOS}

Dermatitis herpetiforme, epidermólisis ampollosa adquirida, dermatosis IgA lineal y lupus ampolloso.

\section{MANEJO}

Se basa en medicamentos inmunosupresores y antinflamatorios. De primera línea están los esteroides, ya sean tópicos en enfermedad leve, o asociados a sistémicos, en enfermedad moderada y grave. También se prefieren algunos antibióticos con efectos antinflamatorios, como las tetraciclinas, específicamente la doxiciclina. De segunda línea también se pueden usar otros medicamentos inmunosupresores, como la azatioprina, el metotrexato, el micofenolato o la dapsona.

Es importante evitar algunos desencadenantes, como los medicamentos y el tratamiento de las enfermedades de base relacionadas.

\section{BIBLIOGRAFIA}

1. Bağcı IS, Horváth ON, Ruzicka T, Sárdy M. Bullous pemphigoid. Autoimmun Rev. 2017;16(5):445-55.doi: 10.1016/j.autrev.2017.03.010.

2. FuertesdeVegaI,Iranzo-FernándezP,Mascaró-Galy JM. Penfigoide ampolloso: guía de manejo práctico. Actas Dermosifiliogr. 2014;105(4):328-46. doi: 10.1016/j.ad.2012.10.022.

3. Velásquez MM, Hoyos JG, Castro LA. Enfermedades ampollosas autoinmunitarias. Rev Asoc Colomb Dermatol. 2012;127-41. 